\title{
ОСОБЕННОСТИ ФОРМИРОВАНИЯ ПРИСПОСОБИТЕЛЬНЫХ РЕАКЦИЙ У ПРИШЛОГО НАСЕЛЕНИЯ КРАЙНЕГО СЕВЕРА
}

\section{FEATURES OF THE FORMATION OF ADAPTIVE REACTIONS IN THE ALIEN POPULATION OF THE FAR NORTH}

\section{A. Shaimardanov}

Summary. The article analyzes the literature on the peculiarities of the formation of adaptive reactions in the alien population of the Far North. The cold climate of the Far North places excessive demands on the mechanisms of adaptation of the human body. Being one of the main adaptogenic factors of the external environment, cold acts as a powerful irritant, therefore, adaptation to cold is considered within the framework of the theory of adaptation to stress. The main mechanisms and types of physiological adaptation to cold are considered. A comparison of various forms of cold adaptation and the associated physiological responses of the body is carried out

Keywords: Far North, cold adaptation, adaptive reactions.

\section{Ввемение}

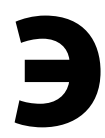
кономика постоянно бросает вызовы человечеству, требуя осваивать новые районы, ранее недоступные для пребывания: это и освоение полярных территорий с целью добычи полезных ископаемых, и расширение зоны расселения людей на планете в связи с ее перенаселением, и освоение морских глубин и космоса. Неадаптированные организмы не могут долго эффективно выполнять работу, находясь в таких условиях. Именно «акклиматизация к определенной среде» дает организму наиболее адекватные преимущества в производительности по сравнению с другими организмами, которые не смогли акклиматизироваться в данной среде [1].

Холодный климат Крайнего Севера предъявляет завышенные требования к механизмам адаптации организма человека. Отрицательное воздействие холода проявляется в различных системах организма и объединяется в понятие «холод-ассоциированные симптомы» $[2,3]$, в которое входят боль, вегетососудистые нарушения, вазоконстрикция, поражения периферической нервной системы, нарушения чувствительности

\author{
Шаймарданов Артур Рифович \\ Аспирант, Сургутский государственный \\ университет \\ dezurengoi@yandex.ru
}

Аннотация. В статье проведен анализ литературы по вопросу особенностей формирования приспособительных реакций у пришлого населения Крайнего Севера. Холодный климат Крайнего Севера предъявляет завышенные требования к механизмам адаптации организма человека. Являясь одним из основных адаптогенных факторов внешней среды, холод выступает, как мощный раздражитель, поэтому, адаптация к холоду рассматривается в рамках теории адаптации к стрессу. Рассмотрены основные механизмы и типы физиологической адаптации к холоду. Проведено сравнение различных форм холодовой адаптации и связанных с ними физиологических ответов организма.

Ключевые слова: Крайний Север, холодовая адаптация, приспособительные реакции.

и изменение цвета открытых частей тела. Холод является одним из основных адаптогенных факторов внешней среды. Поэтому механизмы, которые обеспечивают адаптацию организма к пребыванию в условиях низких температур запускаются даже после кратковременного воздействия холода. При этом происходят изменения, как на уровне всего организма, так и отдельных тканей и органов. Учитывая действие холода не только как специфического раздражителя, способствующего повышению устойчивости гомойотермных организмов к охлаждению, но и как фактора, стимулирующего иммунитет и общую резистентность организма к стрессу [4], различные типы холодовых воздействий с успехом используются для подготовки людей к работе в различных экстремальных условиях [4-6], для повышения функциональных возможностей спортсменов [7, 8], а также в медицине для коррекции и лечения различных заболеваний и травматических повреждений [9-11].

Исследование адаптации пришлого населения крайнего севера ведутся давно, но и до настоящего времени нет единодушия в понимании механизмов ее формирования [12]. 


\section{Це^ь}

Проанализировать литературные данные об особенностях формирования приспособительных реакций у пришлого населения крайнего севера.

Современные методы позволяют изучать внутриклеточные процессы, которые запускаются под действием на организм температурных факторов, рассматриваются различные физиологические механизмы, направленные либо на повышение метаболической мощности и эффективности теплообразования, либо на уменьшение отдачи тепла во внешнюю среду [1214]. Но до сих пор не существует единого объяснения, которое бы учитывало все известные в настоящее время виды адаптации к холоду.

Холод является очень мощным раздражителем. В естественных условиях охлаждение часто носит аварийный характер, и к тому же его действие часто сопряжено с другими стрессорными раздражителями, такими как боль, эмоциональное перенапряжение, гипоксия, повышенная физическая нагрузка и т.д. Поэтому, говоря об адаптации к холоду, следует рассматривать данный процесс в рамках теории адаптации к стрессу.

Согласно теории стресса, которая была предложена Гансом Селье [15], реакция организма на действие экстремальных факторов развивается стадийно и состоит из трех стадий:

1. реакция тревоги (alarm reaction)

2. стадия резистентности (stage of resistence)

3. стадия истощения (stage of exhaustion).

Было обнаружено, что при достаточной силе стрессора даже реакция тревоги может закончиться гибелью организма. Если влияние оказалось меньше летального, то наступает стадия резистентности, которая в случае длительного действия стрессирующего фактора может перейти в стадию истощения.

Еще в 1960-х годах было замечено, что «не все раздражители вызывают однотипную стандартную гормональную реакцию» и «стремление все неспецифические изменения, возникающие в организме, трактовать в качестве проявления стресс-реакции делает это понятие расплывчатым и крайне неопределенным» [16]. В более поздних работах П.Д. Горизонтов [17] разделяет стресс-реакцию на две фазы, где первая является неспецифической и формируется под действием нервной системы, независимо от типа воздействия, а вторая это гормонально зависимый процесс, который отражает специфические особенности воздействующего фактора. И.А. Аршавский [18] выделяет физиологический и патологический стресс, Г.И. Косицким предложено выделять четыре степени напряженности при развитии стресс-реакции [19]. Особый интерес представляет предположение Л.Х. Гаркави с соавторами [20] о существовании неспецифических реакций «тренировки» и «активации». Реакция тренировки развивается в ответ на действие различных по качеству слабых раздражителей, а реакция активации - на действие раздражителей средней силы. Каждая из этих реакций развивается поэтапно и имеет свой комплекс специфических изменений в организме, которые определяют уровень и характер общей резистентности. А стресс-реакция, таким образом, развивается в ответ не на любой, а только на чрезвычайный раздражитель.

Для реакции тренировки характерно колебание содержания лейкоцитов крови и секреции глюкокортикоидов в пределах нормы, в мозге наблюдается доминирование мягкого торможения; активность анаболизма и катаболизма невысокая, с преобладанием анаболизма; процессы накопления энергетических субстратов превышают затраты и создается запас энергии. Биологический смысл реакции тренировки заключается в отсечении слабых, несущественных раздражителей, действие которых повторяется, путем постепенного повышения порога при создании состояния «готовности к защите» [20].

При реакции активации происходит действительное повышение активной резистентности организма за счет усиления его защитных систем. Резистентность при реакции активации не только повышается и поддерживается при повторении активирующих влияний, но, в отличие от реакции тренировки, такая резистентность остается повышенной в течение некоторого времени и после прекращения воздействия раздражителя. Продолжительность данной стадии зависит от возраста и состояния здоровья - повышенная резистентность может сохраняться от 2 недель до полугода.

При реакции активации также не развивается напряжение, как и при стрессе и не преобладают проявления распада в обмене веществ; наоборот, происходит накопление строительного материала - аминокислот, нуклеиновых кислот, белков [20]. Раздражение средней силы является оптимальным для возбуждения защитной функции организма.

Известно, что после первых воздействий холодового фактора развивается острая «аварийная» реакция, направленная на скорую компенсацию функционального состояния организма. Если же холод действует достаточно долго, или его воздействие периодически повторяется, то в организме развиваются устойчивые «физиологические, биохимические, морфологические и поведенческие изменения, которые либо уменьшают 
Таблица 1. Сравнение различных форм холодовой адаптации и связанных с ними физиологических ответов организма

\begin{tabular}{|c|c|c|c|c|c|}
\hline \multirow{2}{*}{ Параметры } & \multicolumn{5}{|c|}{ Типы адаптации к холоду } \\
\hline & M & И & $\Gamma$ & ИГ & Ми \\
\hline $\begin{array}{l}\text { Ректальная } \\
\text { температура } \\
\text { (температура } \\
\text { «дра») }\end{array}$ & $\begin{array}{l}\text { Нормальная } \\
\text { (Не } \\
\text { изменяется) }\end{array}$ & $\begin{array}{l}\text { Нормальная } \\
\text { (Не } \\
\text { изменяется) }\end{array}$ & Снижение & Снижение & $\begin{array}{l}\text { Нормальная } \\
\text { (Не } \\
\text { изменяется) }\end{array}$ \\
\hline $\begin{array}{l}\text { Температура } \\
\text { кожи } \\
\text { (температура } \\
\text { «Оболочки») }\end{array}$ & $\begin{array}{l}\text { Повышение (Вазо- } \\
\text { дилатация) }\end{array}$ & $\begin{array}{l}\text { Снижение (Вазо- } \\
\text { констрикция) }\end{array}$ & $\begin{array}{l}\text { Нормальная } \\
\text { (Не } \\
\text { изменяется) }\end{array}$ & Снижение & $\begin{array}{l}\text { Снижение (Вазо- } \\
\text { констрикция) }\end{array}$ \\
\hline $\begin{array}{l}\text { Метаболическое } \\
\text { продуцирование } \\
\text { тепла }\end{array}$ & Повышение & $\begin{array}{l}\text { Нормальная } \\
\text { (Не } \\
\text { меняется) }\end{array}$ & Снижение & Повышение & Повышение \\
\hline
\end{tabular}

Примечание: МА - Метаболическая адаптация, ИА - Изоляционная адаптация, ГА Гипотермическая адаптация, ИГА - Изоляцийно-гипотермическая адаптация, МИА метаболически-изоляционная адаптация

напряжение, либо повышают выносливость организма к соответствующим изменениям окружающей среды» [21]. Таким образом, в процессе физиологической адаптации к экстремальным условиям, проходит смещение установочной точки (set point), которая характеризует оптимальное функциональное состояние организма в приведенных условиях окружающей среды.

Общепринято, что существует три основных типа адаптации к холоду: это разнообразные адаптации теплообмена (1), изменение поведенческой активности с целью уменьшения влияния холода на организм (2) и миграция в регионы, где для поддержания гомеостаза тратиться меньше энергии (3) [4].

Физиологическая адаптация к холоду связана с двумя основными механизмами:

1 - уменьшение потребления тепловой энергии и ее потери в результате повышения эффективности механизмов изоляции организма (механизмы сохранения тепла);

2 - увеличение производства тепла, которое компенсирует его потерю в окружающую среду.

При определенных условиях указанные механизмы могут действовать одновременно. Механизмы сохранения тепла включают в себя изменения в периферийном кровотоке и противоточный теплообмен между артериальной и венозной кровью в конечностях. Для того, чтобы компенсировать увеличение скорости потери тепла из организма, когда механизмы сохранения тепла не в состоянии поддерживать «нормальную» температуру ядра тела, активизируются метаболические процессы, чтобы генерировать больше тепла, чем при термонейтральный условиях. Важнейшим механизмом создания дополнительного тепла является сократительный термогенез.

В настоящее время принято, что существует несколько видов постоянной адаптации к холоду (гипотермическая, метаболическая и изоляционная адаптации, а также их комбинации) $[12,14,22,23]$. Все эти виды адаптации к холоду были подробно описаны на разных популяциях людей. Холодовая адаптация изучалась у коренного населения в различных регионах планеты, во время полярных экспедиций, у участников зимних спортивных соревнований, у военных и других профессиональных групп, которые часто сталкиваются с холодовым воздействием, а также в контролируемых условиях с влиянием холодного воздуха или воды.

Некоторые авторы предполагают, что указанные виды адаптации к холоду являются отдельными стадиями общего механизма и развиваются в зависимости от силы и продолжительности холодовых воздействий $[1,12,24]$, хотя работ, в которых была бы точно продемонстрирована такая зависимость нами не обнаружено. В то же время имеются наблюдения, одних и тех же 
адаптированных к холоду людей в зависимости от выбранной ими стратегии пребывания под воздействием холода (пассивное или активное), что проявляется реагированием по разным типам (гипотермический или изоляционный соответственно) [25]. Три различных типа (этапа) адаптации к холоду предлагают также K. Brück и P. Hinckel [11]. Они выделяют адаптацию периферических рецепторов (развивается в течение секунд), а также кратковременную (формируется в течение минут) и долговременную (развивается в течение дней и недель) центральную нервную адаптацию.

Согласно A.J. Young гипотермический тип адаптации развивается в ответ на повторяющиеся холодовые воздействия, если состояние для организма нельзя считать опасным, и нет необходимости для формирования сильного термоэфффекторного ответа. Более выраженные физиологические изменения происходят при холодовых воздействиях, если они вызывают значительную потерю тепла: изолирующий тип адаптации к холоду развивается при условиях, когда интенсивность метаболизма оказывается недостаточной, чтобы предотвратить охлаждение ядра тела, а метаболический тип адаптации преобладает, если охлаждение ядра может быть компенсировано увеличением производства тепла [26]. В свою очередь J.H. Bittel [22] предполагает, что тип адаптации к холоду зависит от конституции тела худые люди более склонны к формированию метаболического типа адаптации, чем изоляционного. В пользу этого утверждения свидетельствуют, как отдельные возрастные различия в терморегуляторных ответах на холод, так и половые различия - у мужчин и женщин одного возраста регистрируются различные типы реакции на холодовое раздражение (преобладание сократительного термогенеза у мужчин и несократительного у женщин), что объясняется различным процентным содержанием жировой ткани и скелетных мышц в их организме, хотя скорость снижения температуры ядра тела существенно не отличается [27, 28].

J.P. Cooke со соавторами [29] указывают на различия в поверхностном кровотоке в конечностях мужчин и женщин, что авторы связывают с более частым развитием у женщин, чем у мужчин синдрома Рейно в ответ на действие холода. Некоторые авторы, различия в вазомоторных реакциях, в частности таких как холод-индуцированная вазодилатация сосудов (CIVD), у мужчин и женщин объясняют в первую очередь различием в антропометрических показателях, и для корректности сравнений предлагают использовать данные, полученные для мужчин и женщин одинакового роста и массы тела $[30,31]$. В то же время показано существование двух различных «стратегий» реагирования на холод у неадаптированных мужчин и женщин одного возраста с одинаковой конституцией тела, которые отличались по скорости снижения температуры «ядра» тела [32].

Авторы высказывают предположение, что существование двух различных стратегий реагирования на холод у неадаптированных людей одинакового возраста и телосложения может указывать на существование различных генетических или эпигенетических механизмов, которые запускают эти стратегии. В частности, в работе T. Nishimura с соавторами показано развитие различных типов холодовой акклиматизации в зависимости от наличия определенных митохондриальных гаплогрупп в организме человека [33]. На это могут указывать и данные, согласно которым существует корреляция между некоторыми психологическими особенностями и типом реагирования на холод, в частности экстраверты более выражено реагируют на холодовой дискомфорт, что сопровождается повышением чСС и увеличением секреции норадреналина [34]. Другие авторы $[35,36]$ также указывают на существование не менее двух типов реагирования на острое холодовое влияние в зависимости от особенностей вегетативной регуляции в организме.

Основные различия видов форм холодовой адаптации представлена в таблице 1.

\section{Зак^ючение}

Здоровье людей, осваивающих приполярные и полярные районы, подвергается высокому риску. Приезжие вынуждены адаптироваться к новой среде и, в первую очередь - к холоду. Холод является один из ведущих абиотических факторов Крайнего Севера, способным приводить к истощению компенсаторных и регуляторных механизмов и нарушению постоянства внутренней среды организма. При этом особенности климатических условий северных территорий, несомненно, значительно повышают «цену адаптации», реализация которой не у всех проходит оптимально.

Некомфортные климатические условия Крайнего Севера неблагоприятно воздействуют на функциональное состояние человеческого организма и затрудняют процессы адаптации. При этом реакция адаптации на холодовое воздействие связана, во-первых, с его типом, во-вторых, с интенсивностью, в-третьих, с индивидуальными факторами: возраст, пол, этническая принадлежность, физическая работа на открытом воздухе.

Таким образом, в области физиологии и медицины на Крайнем Севере остается много нерешенных проблем, требующих изучения и дальнейшей разработки. Имеющиеся материалы содержат немало необъясненных фактов или противоречивых данных. 


\section{ЛИТЕРАТУРА}

1. Leppaluoto J., Hassi J. Human physiological adaptations to the arctic climate. Arctic. 1991; 44:139-145.

2. Ульяновская С.А., Баженов Д.В., Шестакова В.Г., Калинкин М.Н. Влияние климатогеографических факторов Севера на адаптивные реакции организма человека. Патол. физиология и эксперим. терапия. 2020; 64; (1): 147-154.

3. Щербакова А.С.Фактор климата в жизнедеятельности северян: объективные данные и субъективные оценки. Экология человека. 2019; $: 24-32$.

4. Пастухов Ю.Ф., Максимов А.Л., Хаскин В.В. Адаптация к холоду и условиям Субарктики: проблемы термофизиологии. Магадан: СВНЦ ДВО РАН; 2003. T. $1,373 \mathrm{C}$.

5. Шевченко В.Е. Медико-биологические причины ухудшения функционального состояния военнослужащих подразделений сил специальных операций В холодных условиях. Вестник проблем биологии и медицины. 2010; (1): 86-90

6. DeGroot D.W., Kenefick R.W., Sawka M.N. Impact of Arm Immersion Cooling During Ranger Training on Exertional Heat Illness and Treatment Costs. Mil Med. 2015; 180(11):1178-83.

7. Janský L., Matoušková E., Vávra V., Vybíral S., Janský P., Jandová D., Knížková l., Kunc P. Thermal, cardiac and adrenergic responses to repeated local. Physiol Res. 2006;55(5):543-9.

8. Lindsay A., Carr S., Cross S., Petersen C., Lewis J.G., Gieseg S.P. The physiological response to cold-water immersion following a mixed martial arts training session. Appl Physiol Nutr Metab. 2017;42(5):529-36.

9. Марченко В.С., Бабийчук В.Г., Ломакин И.И. Механизмы лечебного действия интенсивной криотерапии. Клиническая и экспериментальная патофизиология. 2004; 3 (2): 491-3.

10. Онищенко В.О., Чистилина Е.С., Щеголь М.В. Влияние экстремальной криотерапии на вариабельность сердечного ритма. В: Панченко 0А, редактор. Реабилитация и абилитация человека, клиническая и информационная проблематика. Сборник научн. работ. Киев: КВИЦ; 2012. с. 119-24.

11. Hohenauer E., Costello J.T., Stoop R., Küng U.M., Clarys P., Deliens T., Clijsen R. Cold-water or partial-body cryotherapy? Comparison of physiological responses and recovery following muscle damage. Scand J. Med Sci Sports. 2018;28(3): 1252-62.

12. Castellani J.W., Young A.J. Human physiological responses to cold exposure: Acute responses and acclimatization to prolonged exposure. Auton Neurosci. 2016; 196:63-74.

13. Silva J. Thermogenic mechanisms and their hormonal regulation. Physiol Rev. 2006;86(2):435-64.

14. Makinen T.M. Different types of cold adaptation in humans. Front Biosci (Schol Ed). 2010;2(3):1047-67.

15. Селье Г. Стресс без дистресса. Рига: Виеда; 1992. 109 с.

16. Горизонтов П.Д., Протасова Т.Н. Роль АКТГ и кортикостероидов в патологии (к проблеме стресса). Москва: Медицина; 1968. 334 с.

17. Горизонтов П.Д. Стресс как проблема общей патологии. Вестн. акад. мед. наук. 1979; (11):12-6.

18. Аршавский И.А. Биологические и медицинские аспекты проблемы адаптации и стресс в свете данных физиологии онтогенеза. В: Ливанов МН, редактор. Актуальные вопросы современной физиологии. Москва: Наука; 1976. с. 144-91.

19. Косицкий Г.И., Смирнов В.М. Нервная система и стресс. Москва: Наука; 1970. 260 с.

20. Гаркави Л.Х., Квакина Е.Б., Уколова М.А. Адаптационные реакции и резистентность организма. Ростов-на-Дону: Издательство Ростовского университета; 1990.224 c.

21. Glickman E.L., Cheatham C.C., Caine N., Blegen M. The influence of ethnicity on thermosensitivity during cold water immersion. Aviat Space Environ Med. 2001; 72(7):632-7

22. Bittel J. The different types of general cold adaptation in man. Int J Sports Med. 1992;13 (Suppl 1): S172-6.

23. Daanen H.A.M., Van Marken Lichtenbelt W.D. Human whole body cold adaptation. Temperature. 2016; 3(1):104-18.

24. Brazaitis M., Eimantas N., Daniuseviciute L., Baranauskiene N., Skrodeniene E., Skurvydas A. Time Course of Physiological and Psychological Responses in Humans during a 20-Day Severe-Cold-Acclimation Programme. PLoS One [Internet]. 2014 Apr 10[cited 2015 Mar 09]; 9(4): e94698

25. Hingley E., Morrissey D., Tipton M., House J., Lunt H. Physiology of cold water immersion: a comparison of cold water acclimatised and non-cold water acclimatised participants during static and dynamic immersions. Br J Sports Med. [Internet]. 2011 [cited 2015 Feb19]; 45(2): e1.

26. Young A.J. Homeostatic responses to prolonged cold exposure: human cold acclimatization. In: Fregly M.J., Blatteis C.M., editors. Handbook of Physiology. Section 4: Environmental Physiology, Vol 1. New York: Oxford University Press, 1996. p. 419-38.

27. Solianik R., Skurvydas A., Mickeviciene D., Brazaitis M. Intermittent whole-body cold immersion induces similar thermal stress but different motor and cognitive responses between males and females. Cryobiology. 2014;69(2):323-32.

28. Solianik R., Skurvydas A., Vitkauskienè A., Brazaitis M. Gender-specific cold responses induce a similar body-cooling rate but different neuroendocrine and immune responses. Cryobiology. 2014;69(1):26-33.

29. Cooke J.P., Creager M.A., Osmundson P.J., Shepherd J.T. Sex differences in control of cutaneous blood flow. Circulation. 1990; 82(5):1607-15.

30. Jay 0. , Havenith $\mathrm{G}$. Finger skin cooling on contact with cold materials: an investigation of male and female responses during short-term exposures with a view on hand and finger size. Eur J Appl Physiol. 2004;93(1-2):1-8.

31. Lunt H., Tipton M. Differences in conductive foot cooling: a comparison between males and females. Eur J Appl Physiol. 2014;114(12):2635-44.

32. Brazaitis M., Eimantas N., Daniuseviciute L., Vitkauskiene A., Paulauskas H., Skurvydas A. Two strategies for the acute response to cold exposure but one strategy for the response to heat stress. Int J Hyperthermia. 2015;31(4):325-35 
33. Nishimura T., Motoi M., Niri Y., HoshiY., Kondo R., Watanuki S. Relationship between seasonal cold acclimatization and mtDNA haplogroup in Japanese. J. Physiol Anthropol [Internet]. 2012 Aug 28 [cited 2015 Nov 3];31:22.

34. Sato F., Matsushita S., Hyodo K., Akishima S., Imazuru T., Tokunaga C., Enomoto Y., Kanemoto S., Hiramatsu Y., Sakakibara Y. Sex difference in peripheral arterial response to cold exposure. Circ J. 2008; 72: 1367-72.

35. Mourot L., Bouhaddi M., Regnard J. Effects of the cold pressor test on cardiac autonomic control in normal subjects. Physiol Res. 2009;58(1):83-91.

36. Shattock M.J., Tipton M.J. 'Autonomic Conflict': a different way to die during cold water immersion? J Physiol. 2012;590(14):3219-30.

(c) Шаймарданов Артур Рифович (dezurengoi@yandex.ru ).

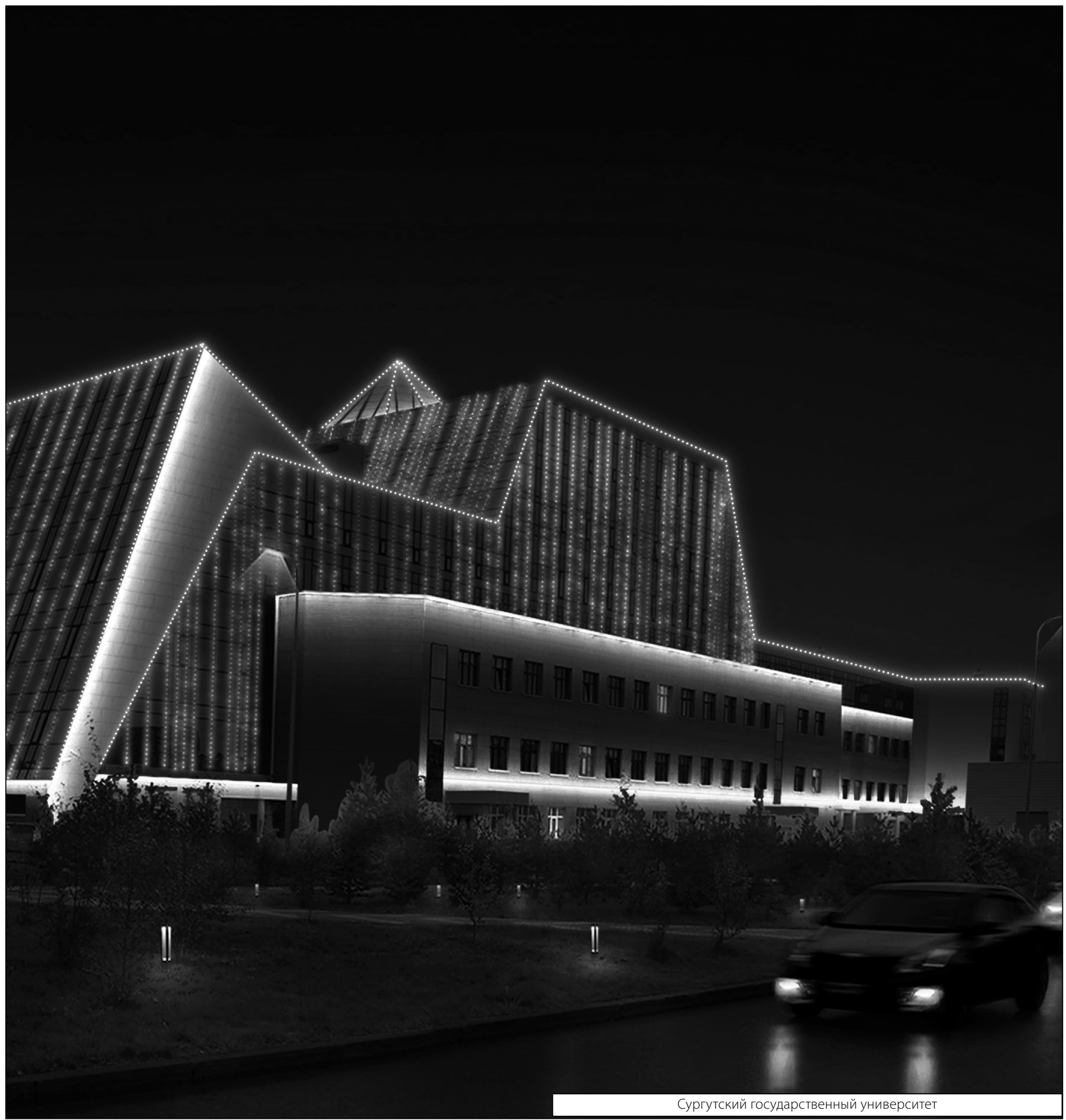

